\title{
Síndrome de Bouveret, una causa extraña de obstrucción intestinal alta: reporte de caso
}

\section{Case report of Bouveret syndrome: a strange cause of upper intestinal obstruction}

\author{
Sergio Andrés Siado ${ }^{1}$, Fermín Alonso Canal ${ }^{1}$, Héctor Conrado Jiménez Sánchez² , Carlos Mauricio Martínez Montalvo ${ }^{3 *}$, \\ Marcela Osorio Santos ${ }^{4}$
}

\footnotetext{
1 Cirujano General Clínica Belo Horizonte Neiva, Huila Colombia.

2 Departamento de Cirugía General, Universidad Surcolombiana, Hospital Universitario Hernando Moncaleano Perdomo, Neiva, Huila, Colombia.

3 Residente Medicina Interna, Universidad del Rosario Bogotá, Colombia.

${ }^{4}$ Médico General, Universidad Pontificia Bolivariana, Medellín, Antioquia, Colombia.

*Correspondencia: Carlos Mauricio Martínez, carlitos220792@gmail.com

Fecha recibido: $\quad 21 / 08 / 18$ Fecha aceptado: $20 / 11 / 18$
}

\begin{abstract}
Resumen
El síndrome de Bouveret (SB) es una patología poco frecuente, la cual se caracteriza por la presencia de obstrucción gástrica o duodenal secundaria a un cálculo enclavado en la luz duodenal, el cual migra a través de una fístula colecistoduodenal. Su incidencia es de alrededor $1 \%$ al $3 \%$ de todos los casos de íleo biliar. Los principales síntomas consisten en vómito, dolor abdominal, hematemesis, pérdida de peso y anorexia. En el $91 \%$ de los casos se requiere manejo quirúrgico. En este artículo se presenta un caso de un paciente de 50 años, con cuadro clínico de 2 meses de evolución de dolor abdominal en epigastrio y mesogastrio, distensión abdominal y múltiples episodios de emesis. Al examen físico con clínica de obstrucción intestinal se realizó tomografía abdominal, donde se evidenció obstrucción intestinal por imagen intraluminal en primera porción duodenal asociada con tríada de Rigler, con diagnóstico de síndrome de Bouveret.
\end{abstract}

Palabras clave

Fístula biliar, síndrome de Bouveret, obstrucción intestinal, íleo biliar.

\begin{abstract}
Bouveret syndrome is a rare pathology which is characterized by gastric or duodenal obstruction secondary to a gallstone embedded in the lumen after migrating through a cholecystoduodenal fistula. Its incidence is approximately $1 \%$ to $3 \%$ of all cases of biliary ileus. The main symptoms consist of vomiting, abdominal pain, hematemesis, weight loss and anorexia. Surgery is required in $91 \%$ of cases. This article presents the case of a 50-year-old patient who had suffered from abdominal pain in the epigastrium and mesogastrium, abdominal distension and multiple episodes of emesis for two months. Physical examination indicated obstruction of the intestine. An abdominal CT scan showed that the obstruction was in the first duodenal portion and that Rigler's triad was present. It was diagnosed as Bouveret Syndrome.
\end{abstract}

Keywords

Biliary fistula, Bouveret syndrome, intestinal obstruction, biliary ileus.

\section{INTRODUCCIÓN}

El síndrome de Bouveret (SB) fue reportado en la literatura desde 1770 por Beaussier y fue nombrado por el médico francés Leon Bouveret, quien, en 1896, realizó dos reportes de caso en la literatura (1). Se define como la presencia de una fístula bilioentérica, la cual permite el paso de cálculos biliares hacia el duodeno, que en su migración proximal hacia el píloro genera una obstrucción del drenaje gastroduodenal $(2,3)$. La fístula es producto de un proceso inflamatorio crónico con aumento de la presión intraluminal, acompañado de isquemia de pared, con un sistema de adherencia y ulterior perforación, lo que conlleva a una comunicación entre el sistema biliar y el intestino, con el paso de cálculos biliares $(4,5)$. 
Representa aproximadamente $1 \%-3 \%$ de los casos de íleo biliar y $2 \%-3 \%$ de los casos obstrucción de intestino delgado $(1,3)$. Su prevalencia es mayor en mujeres y la edad promedio de presentación es de 74 años, con mayor presentación en cálculos $>2,5 \mathrm{~cm}$ o alteraciones posquirúrgicas gastrointestinales; asimismo, actualmente se observa una mortalidad alrededor del 12\% (1-4). En imagenología se caracteriza por la presencia de imagen de cuerpo extraño en la luz duodenal, con o sin cápsula calcificada, asociado con neumobilia y obstrucción intestinal con dilatación duodenal (triada de Rigler) (6-8).

Actualmente, en la literatura se han reportado pocos casos, y aunque su diagnóstico es difícil por la baja especificidad de los síntomas y por tratarse de una patología poco frecuente, las actuales ayudas diagnósticas han permitido tener mayor certeza al momento de diagnosticarlo para poder brindar un manejo oportuno y adecuado de esta patología.

Reportamos el caso de una paciente de 50 años con obstrucción intestinal alta recurrente con manejo quirúrgico inicial (laparotomía exploratoria más liberación de adherencia), secundaria a un SB. Se realiza adicionalmente una revisión de la literatura sobre el tema.

\section{PRESENTACIÓN DE CASO}

Mujer de 50 años con antecedente de apendicetomía 2 años anterior al cuadro clínico y laparotomía exploratoria más liberación de adherencias por obstrucción intestinal secundaria a bridas 2 meses antes de iniciar el cuadro clínico. Consulta refiriendo cuadro clínico de 2 meses de evolución de dolor abdominal tipo cólico a nivel epigástrico referido a mesogastrio, asociado con múltiples episodios eméticos, distensión abdominal, paro de flatos y fecales. Cuadro exacerbado 5 días previos al ingreso.

$\mathrm{Al}$ ingreso, la paciente presenta signos vitales estables, sin signos de respuesta inflamatoria sistémica. Se realiza el examen físico, donde se encuentra abdomen distendido, no doloroso a la palpación, sin presencia de masas o megalias, pero con peristaltismo disminuido. Por lo anterior se consideró cuadro de obstrucción intestinal, por lo cual se inició manejo médico (hidratación endovenosa, sonda nasogástrica de libre drenaje y reposición electrolítica). Se solicitaron paraclínicos de ingreso, los cuales reportan alcalosis metabólica con $\mathrm{pH} 7,53$, hipocalemia de $2,2 \mathrm{mEq} / \mathrm{L}$ y hemograma sin leucocitosis ni neutrofilia (Tabla 1). Se realiza radiografía de abdomen, donde no se evidenciaban signos de obstrucción intestinal, motivo por el cual se solicitó tomografía abdominal (TACab) y endoscopia de vías digestivas altas (EVDA).

En TACab se evidencia obstrucción intestinal por imagen intraluminal a nivel de la primera porción duodenal de $4,5 \times 3 \mathrm{~cm}$ (Figura 1 y 2 ) asociado con neumobilia y pre- sencia de paso del medio de contraste a la vía biliar, lo que cumple los criterios de la triada de Rigler, adicionalmente dilatación de la cámara gástrica secundaria a obstrucción de la luz duodenal y una EVDA con presencia de cálculo impactado en el bulbo duodenal (Figura 3 y 4 ).

Tabla 1. Resultados paraclínicos de ingreso

\begin{tabular}{|c|c|}
\hline $\begin{array}{l}\text { Paraclínicos de } \\
\text { ingreso }\end{array}$ & Resultados \\
\hline Gases arteriales & 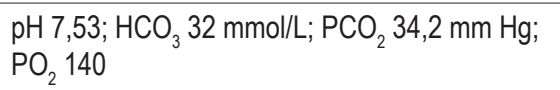 \\
\hline Calcio & $8,7 \mathrm{mEq} / \mathrm{L}$ \\
\hline Sodio & $136 \mathrm{mEq} / \mathrm{L}$ \\
\hline Potasio & $2,2 \mathrm{mEq} / \mathrm{L}$ \\
\hline Hemograma & $\begin{array}{l}\text { Hemoglobina } 12,9 \text {; hematocrito } 36 \text {; leucocitos } \\
7100 \text {; neutrófilos } 62 \% \text {; linfocitos } 22 \% \text {; plaquetas } \\
230000\end{array}$ \\
\hline
\end{tabular}

$\mathrm{HCO}_{3}$ : bicarbonato; $\mathrm{PCO}_{2}$ : presión parcial de dióxido de carbono; $\mathrm{PO}_{2}$ : presión parcial de oxígeno.

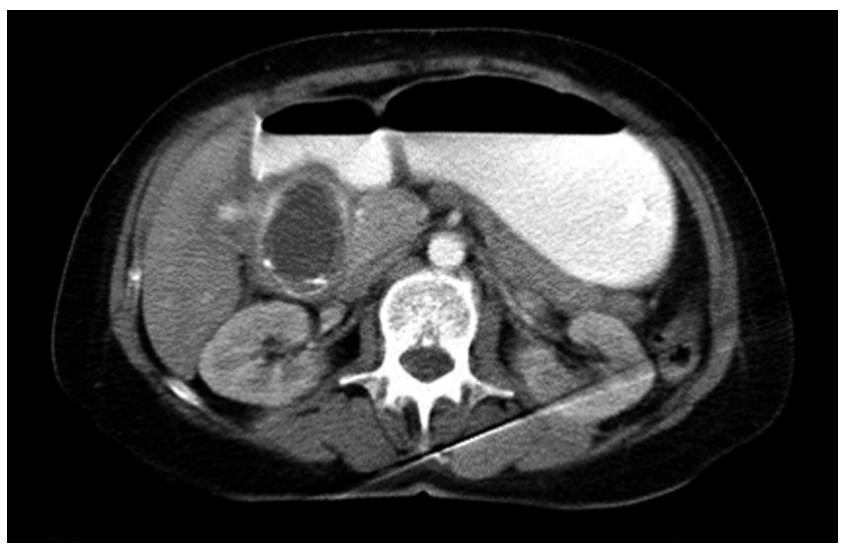

Figura 1. Tomografía axial computarizada de abdomen que evidencia la presencia de imagen redondeada que obstruye la luz duodenal.

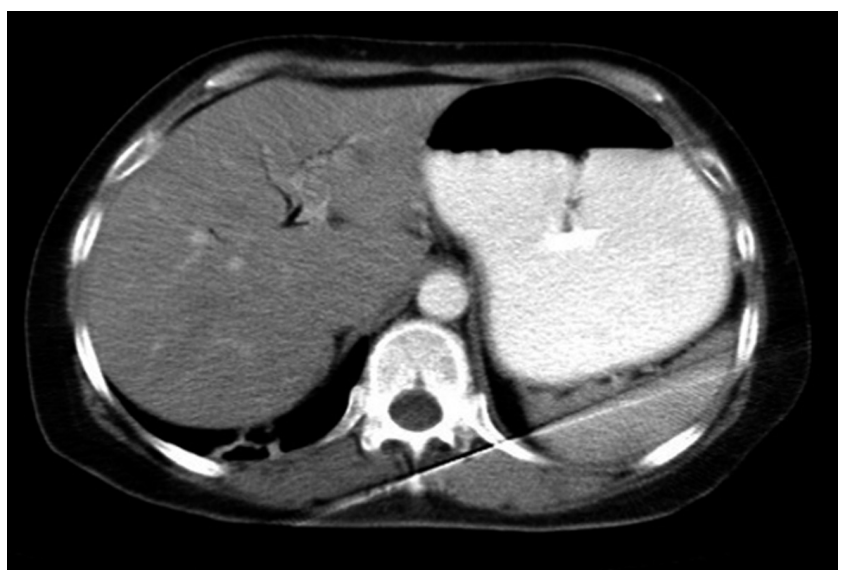

Figura 2. TACab evidencia la presencia de neumobilia y dilatación de la cámara gástrica. 


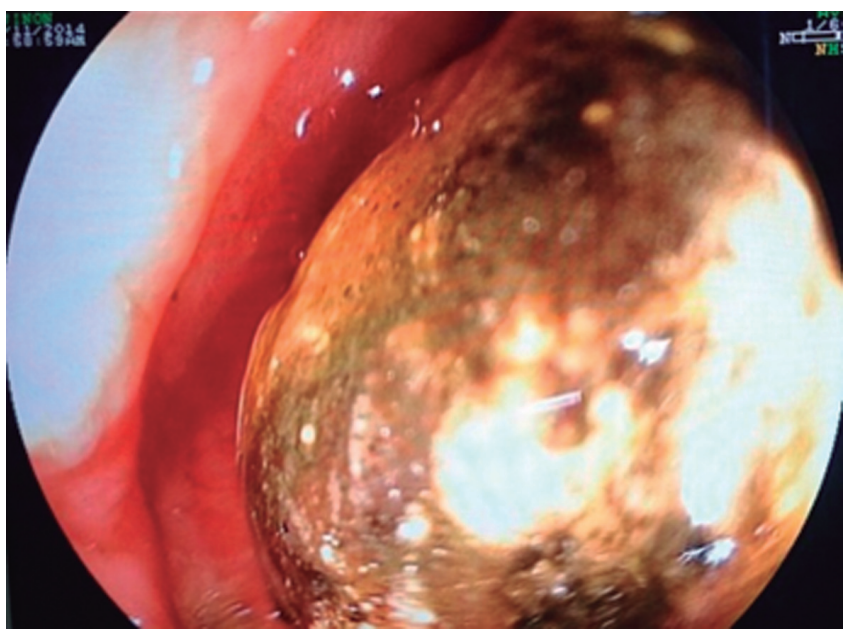

Figura 3. Visión endoscópica de cálculo alojado en la luz del bulbo duodenal.

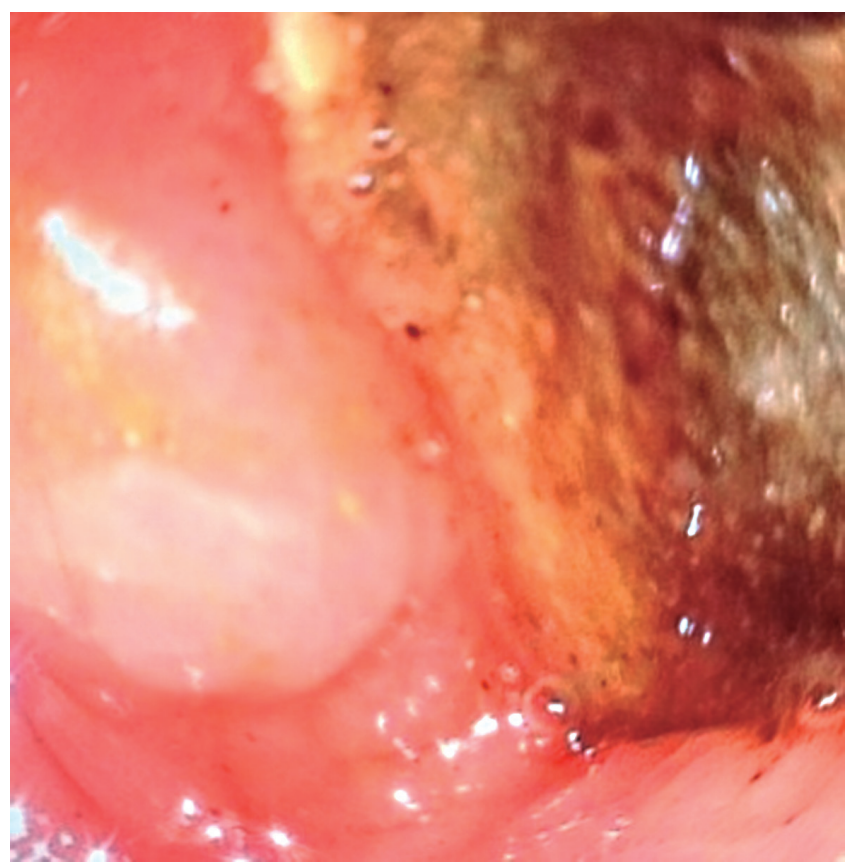

Figura 4. Visión endoscópica de cálculo alojado en la luz del bulbo duodenal.

Dado el cuadro clínico y los hallazgos en las ayudas diagnósticas se consideró cuadro compatible con síndrome de Bouveret y se realizó manejo quirúrgico con laparotomía exploratoria más gastrostomía más extracción de cálculo impactado en bulbo duodenal más gastrorrafía (Figura 5 y 6). Durante el acto quirúrgico se evidencia proceso inflamatorio con múltiples adherencias de asas intestinales y de epiplón a hígado y vesícula biliar, lo cual hizo difícil su visualización.

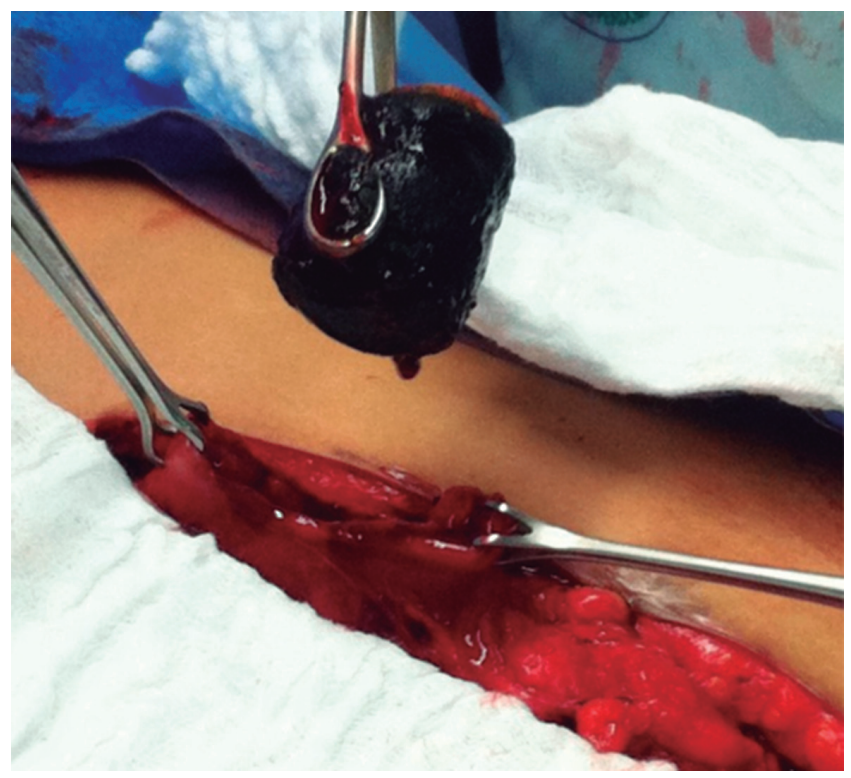

Figura 5. Extracción de cálculo alojado en luz duodenal a través de gastrostomía abierta. En la imagen se evidencia pared anterior de cuerpo gástrico abierta, por la cual se extrae el cálculo.

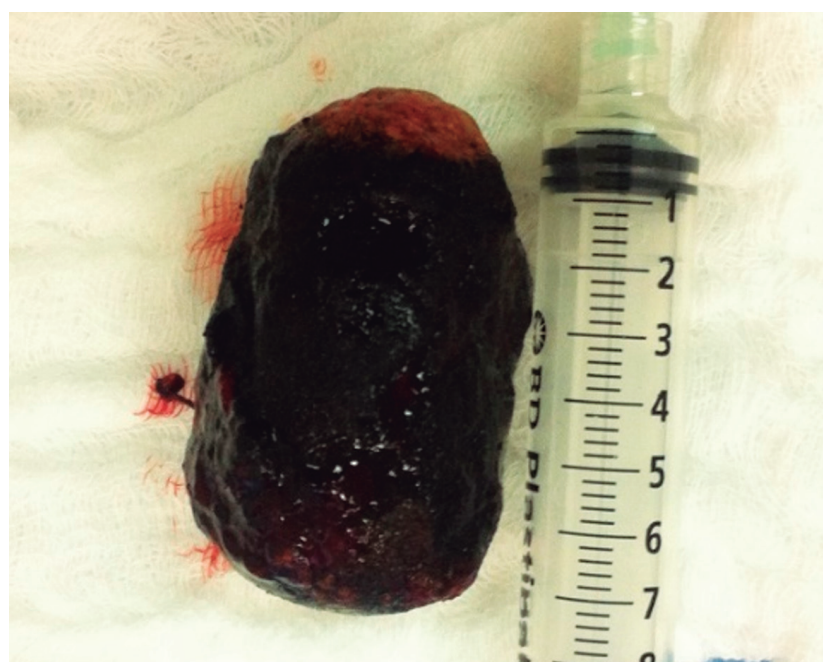

Figura 6. Cálculo biliar extraído de luz duodenal.

Luego, se manejó en el servicio de hospitalización. La paciente presentó evolución satisfactoria y se valoró en control por consulta externa a los 2 meses luego de la cirugía, donde refirió que se encontraba sin dolor abdominal ni nuevos episodios obstructivos, con examen físico dentro de límites normales.

\section{DISCUSIÓN}

El síndrome de Bouveret es una patología poco frecuente, la cual habitualmente se presenta en mujeres de edad avan- 
zada y generalmente con historia de litiasis biliar. Se caracteriza por la presencia de obstrucción gástrica o duodenal, que es secundaria a la impactación de un cálculo en la luz duodenal, el cual migra a través de una fístula colecistoduodenal. Se describió por primera vez en 1896 por Leon Bouveret, sin embargo, solo se presenta en un $15 \%$ de los casos de paso de cálculos al duodeno $(2,8,9)$. Se presenta con clínica inespecífica, como vómito, dolor abdominal, hematemesis, pérdida de peso y anorexia (1). Su incidencia es de alrededor $1 \%$ al 3\% de todos los casos de íleo biliar, por tanto, debido a su baja incidencia requiere un alto índice de sospecha clínica para su diagnóstico.

Con el advenimiento de las ayudas diagnósticas y la disponibilidad de las mismas se ha realizado un adecuado diagnóstico prequirúrgico, siendo la tomografía axial computarizada clave, la cual alcanza una sensibilidad y especificidad de $93 \%$ y $100 \%$, respectivamente, para el diagnóstico. La endoscopia de vías digestivas altas es muy útil en el diagnóstico de esta patología y es considerada el método de referencia para su diagnóstico, como se demostró en el caso reportado, en donde se realizaron los dos exámenes. Las fístulas bilioentéricas pueden variar entre fístula colecistocólica, colecistogástrica o colecistoduodenal, siendo esta última la más frecuente, con $60 \%$ de los casos. Las principales características imagenológicas son presencia de neumobilia, cuerpo extraño alojado en la luz duodenal, obstrucción intestinal con dilatación duodenal y fístula bilioentérica, los cuales concuerdan con la triada de Rigler y se encontraban presentes en nuestra paciente $(9,10)$.

En más del 91\% de los casos, el tratamiento del síndrome de Bouveret requiere manejo quirúrgico, sin embargo, este varía desde el tratamiento endoscópico, que es de primera opción, ya que son pacientes de edad avanzada y con comorbilidades; por tanto, se disminuye el riesgo de morbimortalidad hasta el manejo quirúrgico, ya sea por vía abierta o laparoscópica, mediante enterolitotomía o enterolitotomía con colecistectomía y corrección de la fístula, siendo este último el manejo ideal de los pacientes debido a que disminuye el riesgo de recidivas, hemorragia, colecistitis y cáncer de la vesícula biliar, pero presenta mayores tasas de morbimortalidad (9, 11-14). Es por esto que la elección del manejo varía según las características clínicas y comorbilidades que presenta el paciente, con una mortalidad general de alrededor $12 \%$ al $27 \%$ (2). Para nuestro caso se decidió manejo con enterolitotomía a través de una gastrostomía, siendo las técnicas quirúrgicas más utilizadas en la actualidad, teniendo una adecuada evolución clínica a los 2 meses luego de la cirugía (1).

\section{CONCLUSIÓN}

El síndrome de Bouveret es una patología poco frecuente, de la cual se requiere un alto índice de sospecha clínica para su adecuado diagnóstico. El avance tecnológico de las pruebas diagnósticas ha permitido que su diagnóstico se realice con una alta sensibilidad de manera prequirúrgica, para darle el mejor manejo al paciente de acuerdo con su estado clínico y comorbilidades; por lo anterior, es importante familiarizarse con la semiología radiológica. En nuestro caso, la paciente se manejó con enterolitotomía a través de gastrostomía, siendo la técnica quirúrgica más utilizada en la actualidad, con extracción de cálculo y una evolución satisfactoria a los 2 meses de control.

\section{REFERENCIAS}

1. Haddad FG, Mansour W, Deeb L. Bouveret's syndrome: literature review. Cureus. 2018;10(3):e2299. doi: https:// doi.org/10.7759/cureus.2299.

2. Gajendran M, Muniraj T, Gelrud A. A challenging case of gastric outlet obstruction (Bouveret's syndrome): a case report. J Med Case Rep. 2011;5(1):497. doi: https://doi. org/10.1186/1752-1947-5-497.

3. Ploneda-Valencia CF, Gallo-Morales $\mathrm{M}$, Rinchon C, Navarro-Muñiza E, Bautista-Lópeza CA, de la Cerda-Trujillo LF, et al. Gallstone ileus: an overview of the literature. Rev Gastroenterol Mex. 2017;82(3):248-54. doi: https://doi. org/10.1016/j.rgmx.2016.07.006.

4. Ayantunde AA, Agrawal A. Gallstone ileus: diagnosis and management. World J Surg. 2007;31(6):1292-7. doi: https://doi.org/10.1007/s00268-007-9011-9.

5. Warren DJ, Peck RJ, Majeed AW. Bouveret's syndrome: a case report. J Radiol Case Rep. 2008;2(4):14-7. doi: https://doi.org/10.3941/jrcr.v2i4.60.

6. Rigler LG, Borman CN, Noble JF. Gallstone obstruction: pathogenesis and roentgen manifestations. J Am Med Assoc. 1941;117(21):1753-9. doi: https://doi.org/10.1001/ jama.1941.02820470001001.

7. GanS, Roy-ChoudhuryS, Agrawal S, KumarH, Pallan A, SuperP, et al. More than meets the eye: subtle but important CT findings in Bouveret's syndrome. Am J Roentgenol. 2008;191(1):182-5. doi: https://doi.org/10.2214/AJR.07.3418.

8. Cappell MS, Davis M. Characterization of Bouveret's syndrome: a comprehensive review of 128 cases. Am J Gastroenterol. 2006;101(9):2139-46. doi: https://doi. org/10.1111/j.1572-0241.2006.00645.x.

9. Al-Habbal Y, Ng M, Bird D, McQuillan T, AL-Khaffaf H, et al. Uncommon presentation of a common disease Bouveret's syndrome: a case report and systematic literature review. World J Gastrointest Surg. 2017;9(1):25-36. doi: https://doi.org/10.4240/wjgs.v9.i1.25.

10. Singh AK, Shirkhoda A, Lal N, Sagar P. Bouveret's syndrome: appearance on CT and upper gastrointestinal radiography before and after stone obturation. Am J Roentgenol. 2003;181(3):828-30. doi: https://doi.org/10.2214/ ajr.181.3.1810828.

11. Newton RC, Loizides S, Penney N, Singh KK. Laparoscopic management of Bouveret syndrome. BMJ Case Rep. 
2015;2015:bcr2015209869. doi: https://doi.org/10.1136/ bcr-2015-209869.

12. Alsolaiman MM, Reitz C, Nawras AT, Rodgers JB , Maliakkal BJ. Bouveret's syndrome complicated by distal gallstone ileus after laser lithotripsy using Holmium: YAG laser. BMC Gastroenterol. 2002;2:15. doi: https://doi. org/10.1186/1471-230X-2-15.
13. Caldwell KM, Lee SJ, Leggett PL, Bajwa KS, Mehta SS, Shah SK. Bouveret syndrome: current management strategies. Clin Exp Gastroenterol. 2018;11:69-75. doi: https://doi. org/10.2147/CEG.S132069.

14. Fancellu A, Niolu P, Scanu AM, Feo CF, Ginesu GC, Barmina ML. A rare variant of gallstone ileus: Bouveret's syndrome. J Gastrointest Surg. 2010;14(4):753-5. doi: https://doi.org/10.1007/s11605-009-0918-3. 